DOI: 10.4081/aina.2020.2.78

\title{
COVID-19 pandemic and uro-oncology follow-up: A "virtual" multidisciplinary team strategy and patients' satisfaction assessment
}

\author{
Francesca Ambrosini 1, 2, Andrea Di Stasio 2, Guglielmo Mantica 1, Barbara Cavallone 2, \\ Armando Serao ${ }^{2}$ \\ ${ }^{1}$ Department of Urology, Policlinico San Martino Hospital, University of Genoa, Genoa, Italy; \\ 2 Department of Urology, Azienda Ospedaliera Nazionale SS. Antonio e Biagio e Cesare Arrigo, Alessandria, Italy.
}

\begin{abstract}
Summary COVID-19 pandemic strongly modified the organizations of our clinical practice. Strict containment measures have been adopted to limit the disease diffusion. In particular, hospital face-to-face post discharge and follow up visits have been reduced.

Although cancelling or deferring appointments seems to be a pragmatic approach, this solution may have a devasting long-term impact on health medical care and on patients. In this context, telemedicine and remote consultations may have the potential to provide healthcare minimizing virus exposure.

In this paper we describe how Multidisciplinary team (MDT) reorganized genitourinary cancer care delivery at our Institute (AO SS Antonio e Biagio e Cesare Arrigo, Alessandria), taking advantage of telematic means. Furthermore, we present our preliminary results regarding patients' satisfaction.
\end{abstract}

KEY WORDS: COVID-19; Pandemy; Telemedicine.

Submitted 24 April 2020; Accepted 28 April 2020

In the last few weeks our lives and the organization of our Departments have been completely turned upside down by the coronavirus novel SARS-Cov-2. The virus has rapidly spread all around the world and on the $11^{\text {th }}$ March 2020 the World Health Organization declared the Coronavirus disease 2019 (COVID-19) a global pandemic. Strict containment measures have been taken by governments worldwide in attempt to curb the virus spread, ranging from school closures, social distancing to complete lockdown. As of the $24^{\text {th }}$ April 2020 there have been 2.626.321 confirmed cases with 181.938 deaths and 213 countries involved (1).

COVID-19 is, possibly, the greatest challenge healthcare systems around the world have seen for the last years, since drastic health resources reallocations have been necessary to face the pandemic. In this context, routine clinical and surgical activities, including urological practice, have been deeply reorganized prioritizing patients' safety and well-being. Recommendations on the reorganization of urological activities have been provided by different scientific societies (2). In particular, all elective surgeries should be deferred, and surgical intervention should be limited for high grade malignancies and unstable trauma patients. Hospital face-to-face post discharge and follow up visits should be reduced implementing telehealth strategies.

We believe that oncological communications are by far the most delicate ones to share with the patient and that deserve particular attention.

Before COVID-19 pandemic, weekly multidisciplinary hospital follow-up visits were carried out to patients treated for genitourinary cancers at our Institute. Our local Multidisciplinary Team (MDT) involves medical oncologists, radiotherapists, pathologists, clinical nurse specialists and urologists. In order to reduce home-hospital flow, all patients scheduled from March $9^{\text {th }} 2020$, first day of national lockdown, onwards have been phone called by the clinical nurse specialist and their email collected. They were asked to send the results of their instrumental and biochemical exams' follow up by mail and to communicate any new clinical onset problem. The Patients' documents and data were collected and then examined by MDT members on weekly videoconference meetings through the use of Zoom (Zoom Video Communications, Inc. San Jose, California, 2011).

A clinical report containing oncological outcomes and the corresponding planning, with follow-up exam and visit, were sent to the patients via e- mail accompanied by a telephone call from the urologist. Face to face consultations were carried out only in case of disease progression or recurrence, onset of metastasis or severe clinical troubles.

The follow-up of 56/60 (93.3\%) scheduled patients were successfully managed by the "virtual" MDT, while only 4 patients required a conventional outpatient clinic setting. No complaints, objections or criticisms were recorded, regarding the proposed remote monitoring. At the end of the "virtual" consultations, the patients were asked to reply an anonymous online survey through Google Forms.

With a response rate of $68.3 \%(41 / 60)$ most of the patients showed a high satisfaction (mean: 4.7/5) with no serious complaining about major technical issues. The smartness and convenience of the "virtual" visits were indicated as the most appreciated advantage by 10 
(24.4\%) and 16 (39\%) patients, respectively. The lack of Clinicians' physical presence was perceived as the main limit of tele-visit by eleven patients (26.8\%).

Furthermore, 38/41 (92.7\%) of the patients felt adequate sensitive data protection and no significant concerns about the privacy and security of remote visits.

To date, while telemedicine is gaining popularity and numerous platforms and apps are available, it still poses some technical and practical problems. One of the main limits seems to be absence or unclearness of the legislation that still remains a common stumbling block for providers and patients (3).

Telemedicine may increase clinical workflow efficiency in the coming years offering several benefits to the health system organization.

SARS-Cov-2 pandemic is now driving the whole word transformation and could provide the decisive push to make institutions, clinicians and, above all, patients familiar with telemedicine.

\section{References}

1. Coronavirus [Internet]. World Health Organization. 2020 [cited 24th April 2020]. Available from: https://www.who.int/emergencies/ diseases/novel-coronavirus-2019.

2. Leonardi R, Bellinzoni P, Broglia L, et al. Hospital care in Departments defined as COVID-free: A proposal for a safe hospitalization protecting healthcare professionals and patients not affected by COVID-19. Arch Ital Urol Androl. 2020; 92(1). https://doi.org/ 10.4081/aiua.2020.2.67.

3. Castaneda P, Ellimoottil C. Current Use of Telehealth in Urology: A Review. World J Urol. 2019 Jul 27. doi: 10.1007/s00345-01902882-9. Online ahead of print.

\section{Correspondence}

Francesca Ambrosini, MD (Corresponding Author)

f.ambrosinil@gmail.com

Department of Urology, Policlinico San Martino Hospital, University of Genoa

Largo Rosanna Benzi, 10, 16132, Genoa, Italy

Department of Urology, Azienda Ospedaliera Nazionale SS. Antonio e Biagio e Cesare Arrigo

Via Venezia 16, 15121, Alessandria, Italy

ORCID 0000-0003-2160-763X

Andrea Di Stasio, MD

andrea.distasio@libero.it

Barbara Cavallone, MD

barbara.cavallone@ospedale.al.it

Armando Serao, MD

aserao@ospedale.al.it

Department of Urology, Azienda Ospedaliera Nazionale SS. Antonio e Biagio e Cesare Arrigo, Alessandria (Italy)

Guglielmo Mantica, MD

guglielmo.mantica@gmail.com

Department of Urology, Policlinico San Martino Hospital, University of Genoa, Genoa, Italy 